\title{
Fabrication and Characterization of 3D Printed Thin Plates for Acoustic Metamaterials Applications
}

\author{
Cecilia Casarini, Student Member, IEEE, Vicent Romero-García, Jean-Philippe Groby, Ben Tiller, \\ James F. C. Windmill, Senior Member, IEEE and Joseph C. Jackson
}

\begin{abstract}
This paper presents a 3D printing technique based on stereolithography and direct light processing for the fabrication of low resonance frequency thin plates suitable for acoustic metamaterials applications. It was possible to achieve a better resolution with respect to other $3 \mathrm{D}$ printing methods such as fusion deposition modeling and to obtain plates with a thickness of $70 \mu \mathrm{m}$. The plates were characterized using three different methods: laser Doppler vibrometer supported by modal analysis, impedance tube measurements backed by a transfer matrix model and nanoindentation. All results are in good agreement. The physical parameters retrieved through the characterization methods can be used for future designs and integrated into finite element analysis to better predict the noise impact of these materials. Thanks to the small radius and thickness of the plates presented in this paper and to their low resonance frequency, it is suggested that they could be arranged in various configurations and used as unit cells in acoustic metamaterials applications for noise attenuation in smallscale electroacoustic devices.
\end{abstract}

Index Terms-Acoustic Metamaterials - Thin Plates Membranes - 3D Printing - Characterization - Noise Attenuation

$\begin{array}{ll}\text { Nomenclature } & \\ \text { PEGDA } & \text { Polyethylene Glycol Diacrylate } \\ \text { BaTiO3 } & \text { Barium Titanate } \\ \text { SI } & \text { Sudan I } \\ \text { I819 } & \text { Irgacure } 819 \\ \mathrm{MW} & \text { Molecular Weight } \\ \mathrm{LDV} & \text { Laser Doppler Vibrometer } \\ E & \text { Young's Modulus } \\ \rho & \text { Density } \\ v & \text { Poisson's Ratio } \\ \eta & \text { Loss Factor } \\ \mathrm{f}_{0} & \text { Fundamental Frequency }\end{array}$

Manuscript submitted May 1, 2019. This research is funded by the European Research Council under the European Union's Seventh Framework Programme (FP/2007-2013) / ERC Grant Agreement n. [615030] and by COST Action DENORMS CA15125, supported by COST (European Cooperation in Science and Technology).

Cecilia Casarini, Ben Tiller, James F. C. Windmill and Joseph C. Jackson are members of the Centre for Ultrasonic Engineering, Electronic and Electrical

\section{INTRODUCTION}

$\mathrm{R}$ ecent advances in additive manufacturing techniques have now made it possible to fabricate thin membranes and plates that are essential for the study of several acoustic applications - for example, microphones and loudspeaker prototyping and acoustic metamaterials [1]-[4]. In laboratories, these membranes are usually obtained through a procedure where a piece of material is cut and glued on a rigid frame or support. On the other hand, industrial fabrication typically relies on other techniques - such as clean room manufacturing - that have high maintenance costs. In comparison with these established production processes, additive manufacturing provides with a cheap and reliable fabrication approach, which additionally offers the opportunity to choose the material for the membrane. Furthermore, 3D printing software allows to create abstract shapes and to integrate the product inside other structures, for example by 3D printing a membrane inside a Helmholtz resonator. Haque et al. [2] fabricated a thin silver layer on a purchased Mylar film using a inkjet 2D printer, that deposits drops of liquid material on pre-existing substrate. Previous research on 3D printing membranes relied on fusion deposition modeling [3], which deposits a filament layer by layer through a heated extruder nozzle head. Stereolithography via direct light processing has recently been used to $3 \mathrm{D}$ print thin layers with thickness down to $35 \mu \mathrm{m}$ [1], thanks to new techniques that rely on absorbers that allow to control shapes and thickness of the materials in a finer way with respect to fusion deposition modelling, that has a minimum thickness of $0.1 \mathrm{~mm}$. The advantages of these new stereolithography techniques can be found not only in the practical utility of fabricating a thin layer, but also in the opportunity to finely control its material. Moreover, multilayer plates made of different materials can be fabricated, paving the way of manufacturing a sensor or even an entire device in one print [1], [2]. In particular, when using polymers it is possible to choose the desired physical properties of the plate, given the vast range of different options available within the polymers

Engineering Department, University of Strathclyde, Glasgow, Scotland, UK. email: cecilia.casarini@strath.ac.uk.

Vicent Romero-García and Jean-Philippe Groby are members of the Laboratoire d'Acoustique de l'Université du Maine (LAUM), Le Mans, France. 
realm [1], [5]. In this work, polymer-based thin low-frequency circular acoustic plates are fabricated using stereolithography and experimentally characterized via three different techniques.

Although the fabrication technique described in this paper could be useful for many different applications based on membranes and plates, e.g. in microphones manufacturing [1] and non-destructive evaluation [6], the design presented here is specifically tailored to noise control through acoustic metamaterials. Plate-type acoustic metamaterials, originally proposed by Yang et al. [7]-[9], present a new and promising solution to the problem of low-frequency noise attenuation. Their small thickness and light weight could make them more desirable, for certain applications, than traditional materials used for noise control. Plates with or without a mass attached present a region of negative effective density with high sound transmission loss between the first and the second resonance, when backed with a rigid cavity, while plates suspended on an open support - as the ones presented in this paper - show this behavior in the frequency band preceding the first mode [10][16]. Thin membranes and plates can be used as unit cells in acoustic metamaterials presenting different configurations. For example Yang et al. [9] arranged them in in a panel, Sui et al. [16] sandwiched the membranes between honeycomb panels and Naify et al. [17] built stacked membranes arrays. The thin plate presented in this paper could be arranged in any of the configurations described above. Furthermore, the fabrication technique presented here provides the opportunity to directly 3D print and integrate the plates inside other complex unit cells such as Helmholtz resonators and pipes to broaden their band gap. Being able to control the thickness, radius and material properties of the plates through additive manufacturing allows to choose the best combination of parameters to match a specific frequency range to be attenuated, while respecting the dimension constraints of a specific application. For example, in this paper the goal is to fabricate thin plates that could be included in small-scale devices, such as headphones and smart speakers, and at the same time having a low resonance frequency. Therefore, a polymer that had a low Young's modulus with respect to other materials used in previous work [4] was selected. The 3D printing technique here developed made it possible to fabricate layers $70 \mu \mathrm{m}$ thick. The chosen materials allowed to obtain a small-scale thin plate with a low resonance frequency.

Characterization is a crucial step in the production process to predict the acoustic behavior of a device at the design stage. This is particularly true for 3D printing applications, where the physical quantities of the materials change throughout the polymerization process. A good estimate of properties such as Young's modulus, Poisson's ratio, density and loss factor is very important to design more complicated devices that include these plates and to predict their acoustic impact before fabrication using finite element analysis or other modeling techniques. In the following sections the fabrication process used to 3D print the plates is first described in detail, then, three different characterization methods are introduced. Laser Doppler vibrometer is used to show that the 3D printed layer presents the typical modes of vibration found in circular plates and membranes. Impedance tube measurements assessed by the transfer matrix modeling approach are employed to retrieve the main physical parameters such as Young's modulus, density, Poisson's ratio and loss factor, and to obtain the acoustic coefficients, namely absorption, transmission and reflection. Finally, nanoindentation together with scanning electron microscopy give further insight on the properties of the plate. Future work and potential industrial applications are outlined in the Conclusion section.

\section{MATERIALS AND METHODS}

\section{A. Plates Fabrication}

This section firstly presents the design choices in terms of geometry and materials and secondly describes in detail the thin plate fabrication steps. An Asiga Pico Plus 27 (Sidney, Australia) with a pixel image resolution of 27 by $27 \mu \mathrm{m}$, and a minimum build layer thickness of $1 \mu \mathrm{m}$ was used to $3 \mathrm{D}$ print the samples. A circular shape was selected for the plate, since the analytical model of circular membranes and thin plates is well known and also due to previous research based on cylindrical Helmholtz resonators [4] that could be integrated with thin plates in future work. A hollow cylinder $1 \mathrm{~mm}$ in height and with $1 \mathrm{~mm}$ thick walls was also designed to support the plate. The 3D printer build plate size is $33.49 \times 20.99 \times 76$ $\mathrm{mm}$ and the outer diameters of the support and plate were set at $20 \mathrm{~mm}$, which is the maximum diameter. Therefore, the effective diameter of the membrane was $15 \mathrm{~mm}$. There are two main reasons for selecting the maximum possible diameter. Firstly, in order to characterize the plate with an impedance tube, the diameter should be as large as possible, given the standard dimension of the tube, in this case having a $30 \mathrm{~mm}$ diameter. Secondly, since the resonance of a thin plate is inversely proportional to the square of its radius, a larger diameter would result in a lower frequency, as required for noise applications.

As shown in Figure 1, two different samples were fabricated. The first sample had as main component polyethylene glycol diacrylate 250 (PEGDA 250) and the second polyethylene glycol diacrylate 700 (PEGDA 700). The numbers 250 and 700 represent the molecular weights (MW) of the two different types of PEGDA. In particular, PEGDA 700 was preferred for this work as it is characterized by a lower Young's modulus than PEGDA 250 and hence a lower resonance frequency, that could match and attenuate lower frequencies. BaTiO3 nanopowder with a $500 \mathrm{~nm}$ diameter average particle size, purchased from US-NANO, was added to the resin with a 50\% weight with respect to PEGDA when fabricating the plate. $\mathrm{BaTiO} 3$ main function was to scatter the light in a uniform way on the horizontal plane, to obtain a flat plate, since previous attempts that did not include $\mathrm{BaTiO} 3$ resulted in a curved plate. It is important to notice that by adding $\mathrm{BaTiO} 3$ to the resin, a piezoelectric material can be obtained [1] and it could be used as such in future implementation of this work. Irgacure 819 (I819) was added to both mixtures as a photoinitiator with a $1 \%$ weight with respect to PEGDA and $0.1 \%$ Sudan I (SI) was added as absorber to better control the amount of light that could contribute to the polymerization of the resin. These chemicals were purchased from Sigma Aldrich. While the resin described above was used to print the thin plate, the support material was made of PEGDA 250, 0.1\% Sudan I and 1\% 
Irgacure 819. The exposure time to light contributes to determining the thickness of the membrane [18] and different exposure times between 0.5 second and 9 seconds where tried for different materials as reported in [4]. Since the Asiga 3D printer software does not allow to change the exposure time to light or other parameters while the print has started, the chosen exposure time of 5 seconds was of a duration that allowed to print both the materials of the support and the materials of the membrane.

The first step in the fabrication process, as shown in Figure 2, consisted in mixing the chemicals to obtain the resin for the thin plate and the resin for the support. The two resins were then independently mixed for 2 minutes using Thinky ARE 250 planetary mixer (INTERTRONICS) and then sonicated for 15 minutes. The CAD design was then uploaded into the Asiga Composer 3D Printing Software and automatically sliced into 101 layers, each $10 \mu \mathrm{m}$ thick. The Layers 1 to 100 corresponded to the support and layer 101 to the thin plate. The resin for the support was then poured into the build tray and the $3 \mathrm{D}$ printing process was started. The support and the plate were printed in one piece, by pausing the $3 \mathrm{D}$ printer in correspondence to the layer before the plate, i.e. layer 100 , leaving the 3D printed PEGDA part attached to the build platform to keep the right calibration. After cleaning the part on the build platform with isopropyl alcohol (IPA), the liquid resin in the build tray was changed to the resin of the thin plate and the last layer was fabricated. It is important to notice that, while the chosen layer thickness is $10 \mu \mathrm{m}$, when printing on a void the final thickness is determined by the intensity of light, the absorption coefficient of the fluid and the exposure time to light [18]. With commercial resins, even when the build layer thickness was set to $10 \mu \mathrm{m}$ or less, the plates resulted to be $1 \mathrm{~mm}$ thick. As shown in Figure 3, when adding SI to the resin to reduce the amount of light, it was instead possible to finely control the resolution and to obtain a thickness down to $35 \mu \mathrm{m}$ [1], [4]. In this case, the fabricated plate had a thickness of $70 \mu \mathrm{m}$, which was measured using a digital micrometer. Panel (a) of Figure 3 shows the 3D printing process of the support first 100 layers. When the last layer is fabricated (panel (b)), the part of fluid that polymerizes underneath the air cavity expands beyond the expected position in the $\mathrm{z}$ axis, resulting in a thicker membrane. When SI is added to the resin (panel (c)), the polymerization process can be finely controlled, and the last layer corresponds to the selected thickness.

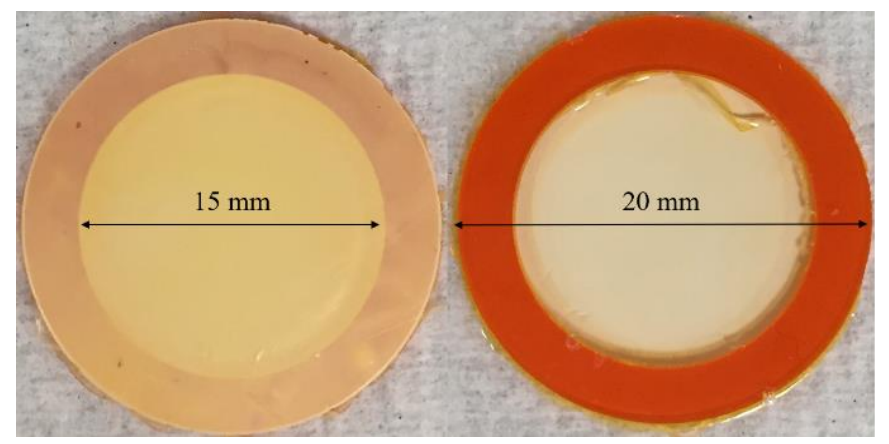

Fig. 1. 3D printed plates front (left) and back (right).

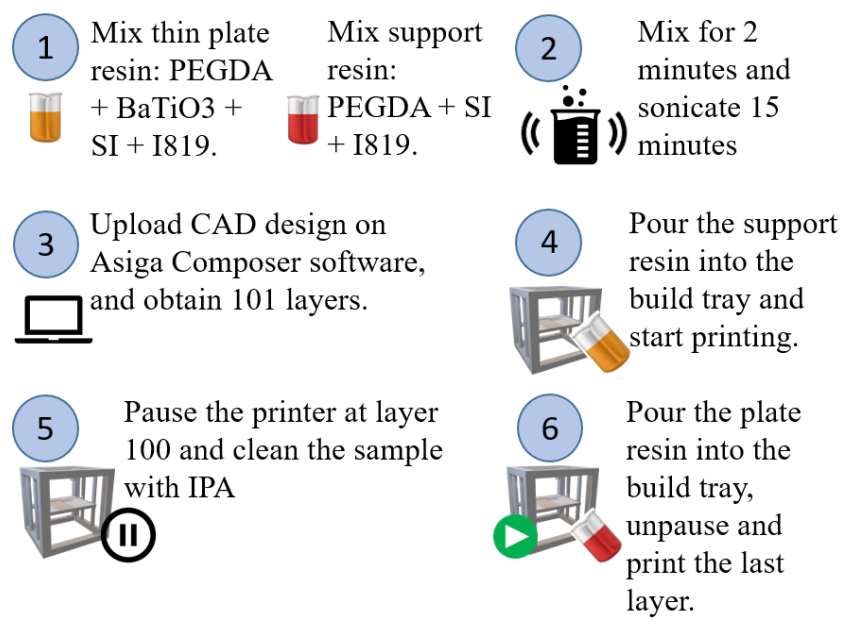

Fig. 2. Diagram representing the 3D printing process step by step.

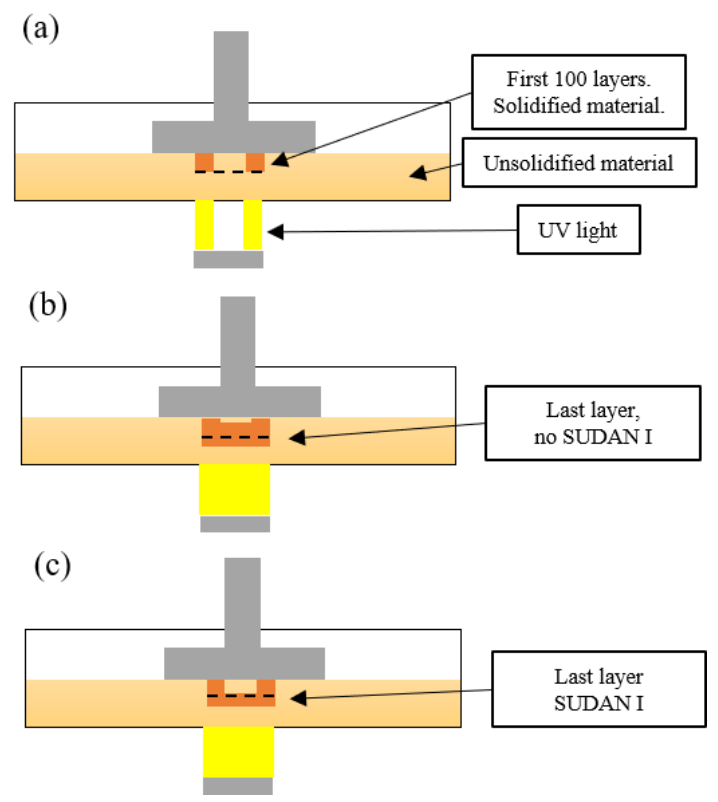

Fig. 3. The first 100 layers of the sample, i.e. the empty cylindrical support, are first 3D printed thanks to the projection of the UV light according to the selected geometry and to the consequent polymerization of the liquid material contained in the build tray (a). When no SUDAN I is added to the liquid resin, the last layer, i.e., the plate, polymerizes beyond its defined thickness, expanding in the void inside the support (b). Adding SUDAN I allows to better control the amount of light absorbed by the resin and to $3 \mathrm{D}$ print a plate with the correct thickness (c).

\section{B. Characterization Using Laser Doppler Vibrometer}

A Polytec 3D scanning laser Doppler vibrometer (LDV) was used to perform the first characterization of the modes and fundamental resonance of the plates under test. Each sample was fixed to a glass support, and a loudspeaker (ESS Heil AirMotion Transformer) was used to create a sweep between 100 and $20000 \mathrm{~Hz}$. The vibration of the plate was mapped by 289 scanning points. The Polytec software was used to analyze the results and plot the vibration modes of the plate under test. 


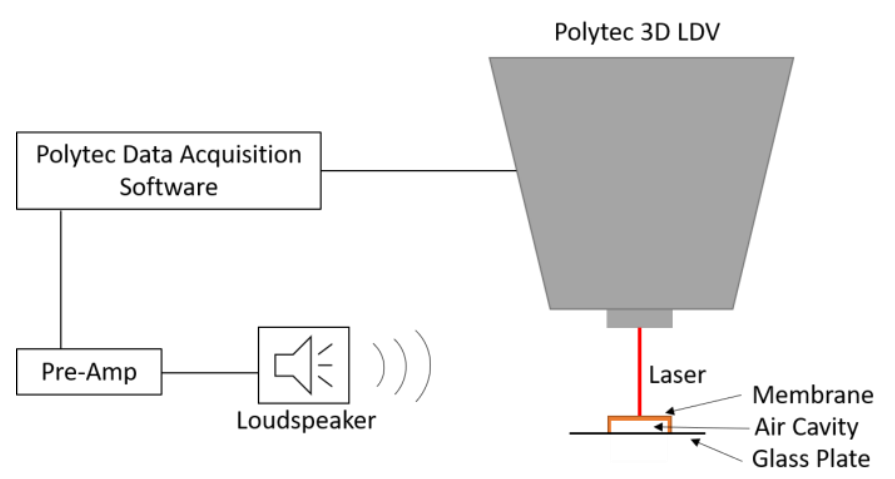

Fig. 4. Experimental setup used during data acquisition with a Polytec laser Doppler vibrometer (LDV). The vibration modes of the sample were excited acoustically though a sine sweep.

\section{Transfer Matrix Model and Characterization Using Impedance Tube}

To acoustically characterize the plates, a $30 \mathrm{~mm}$ in diameter impedance tube (CTTM, Le Mans, France) represented in Figure 5 and shown in Figure 6 (a) was used to experimentally measure the transmission, reflection and absorption coefficients. A brass sample holder shown in Figure 6 (b) and (c) to be inserted into the tube was also fabricated. The sample holder consists of two hollow cylinders, the first one having a cavity where the plate sample can be fit. Four joints extrude from the second cylinder and secure the connection with the first cylinder that presents four matched hollows. The plate is inserted into the first cylinder and then the second cylinder is connected and simply secured. The system was modeled through a transfer matrix approach [19]. The parameters assumed in the model were then optimized by fitting the measurements results into the model [20].

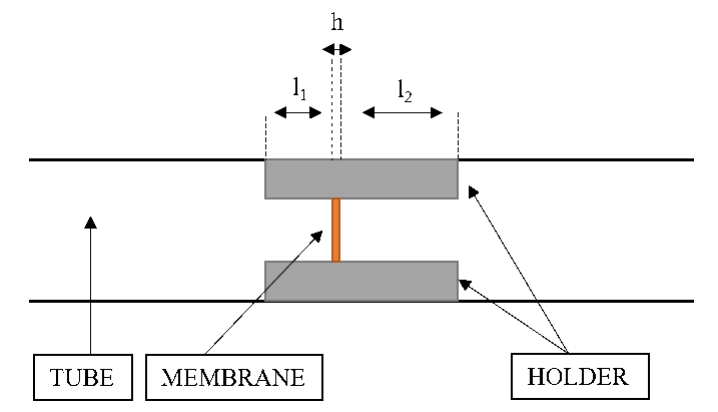

Fig. 5. Scheme of the system modeled through the transfer matrix method. As in the experiments, the impedance tube contains a sample holder that supports the plate.

As shown in Figure 5, the system to be modeled consisted of the plate with thickness $h$, the sample holder modeled as two air cylinders of length $l_{1}$ and $l_{2}$ on both sides of the plate and their end corrections. The sample holder is modeled as a circular tube and its wave number $k_{t}$ and the impedance $Z_{t}$ are derived as in [21]. Therefore, the transfer matrix $T=\left(\begin{array}{ll}T_{11} & T_{12} \\ T_{21} & T_{22}\end{array}\right)$, as discussed in [19], [22], is equal to:

$T=M_{\text {endcorr }} M_{\text {tube } 1} M_{\text {mem }} M_{\text {tube } 2} M_{\text {endcorr }}$, where $M_{\text {endcorr }}$ is the transfer matrix of the end correction $\Delta l[23]:$

$M_{\text {endcorr }}=\left(\begin{array}{cc}1 & \mathrm{i} Z_{t} k_{t} \Delta l \\ 0 & 1\end{array}\right)$.

$M_{\text {tubej }}$ is the transfer matrix of the sample holder on the left ( $\mathrm{j}$ $=1)$ and right $(\mathrm{j}=2)$ sides of the plate [24]:

$M_{\text {tubej }}=\left(\begin{array}{cc}\cos \left(k_{t} l_{j}\right) & \mathrm{i} Z_{t} \sin \left(k_{t} l_{j}\right) \\ \frac{i \sin \left(k_{t} l_{j}\right)}{z_{t}} & \cos \left(k_{t} l_{j}\right)\end{array}\right)$.

$M_{\text {mem }}=\left(\begin{array}{cc}1 & Z_{m} \\ 0 & 1\end{array}\right)$

where $Z_{m}$ is the acoustic impedance of the thin plate [25]:

$Z_{m}=-\mathrm{i} \omega m \cdot \frac{I_{1}\left(k_{m} a\right) J_{0}\left(k_{m} a\right)+\left(J_{1} k_{m} a\right) I_{0}\left(k_{m} a\right)}{I_{1}\left(k_{m} a\right) J_{2}\left(k_{m} a\right)-J_{1}\left(k_{m} a\right) I_{2}\left(k_{m} a\right)}$

$k_{m}$ is derived in [25] $a$ is the radius of the plate, $J_{n}$ and $I_{n}$ are respectively regular and modified Bessel's functions of the first kind of order $n$ and $m$ is the mass of the plate. The wave number of the plate $k_{m}$ is equal to [25]:

$k_{m}^{2}=2 \pi f \sqrt{\frac{\rho \prime^{12\left(1-v^{2}\right)}}{E h^{3}}}$,

where $\rho_{m}^{\prime \prime}=\rho_{m} h$ is the surface mass density of the plate with mass density $\rho_{m}=\frac{m}{S h}$, where $m$ is the mass of the plate and $S$ its cross-section. The resonance frequency $f_{0}$ is given in terms of the the Young's Modulus $E$, Poisson's Ratio $v$ and loss factor $\eta$ and the geometry by [26]:

$f_{0}=0.4694 \frac{h}{a^{2}} \sqrt{\frac{E}{\rho_{m}\left(1-v^{2}\right)}}$.

Transmission, reflection and absorption coefficients can be retrieved from the total transfer matrix $T$ according to [27], [28].

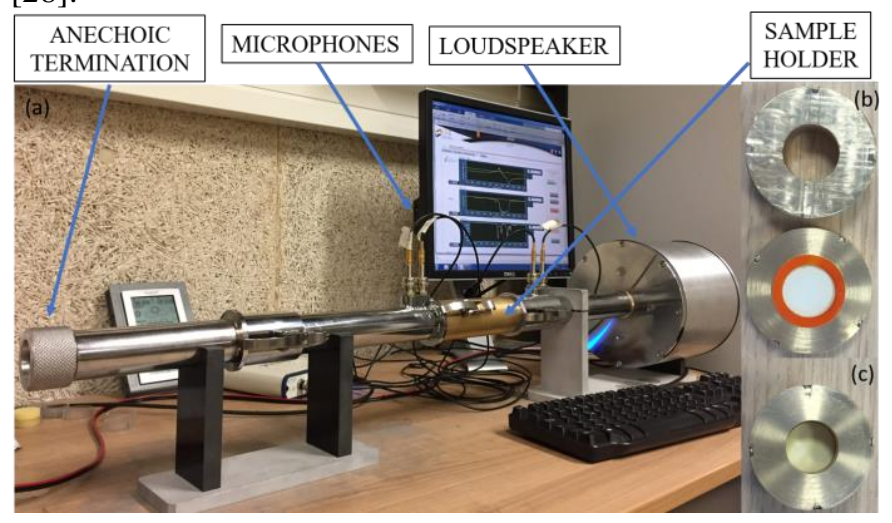

Fig. 6. Impedance tube used to measure the acoustic coefficients of the plate under test (a). Open sample holder with plate inserted (b) and closed sample holder with plate (c). 
The impedance tube used to measure the acoustic coefficients had four microphones, an anechoic termination on one side and a loudspeaker on the other termination, as illustrated in Figure 6 (a). A sine sweep between $20 \mathrm{~Hz}$ and $2500 \mathrm{~Hz}$ was used as input [29]. Each sample was measured 10 times to assess the repeatability of the measurements. The reflection, transmission and absorption coefficients were retrieved from the measurements [19], [28]. Then, the physical quantities, chosen to initialize the model, namely density, Poisson's ratio loss factor and Young's modulus, were optimized to fit the experiments.

\section{Characterization Using Nanoindentation}

In order to compare different characterization methods and to determine which one is most suitable for this kind of samples, a 3D nanoindenter was also used to retrieve the Young's modulus of the plate. The 3D printed plate was cut and just a small sample of it was used during this process. A Berkovich tip made of single crystalline diamond was used for nanoindentation and a IBIS software retrieved the Young's Modulus of the materials. The values of the reduced elastic modulus were determined using the method of Oliver and Pharr [30]. The Young's modulus was then obtained from the reduced Elastic modulus assuming a Poisson's ratio of 0.35 . To better understand if this characterization process was compatible with the $3 \mathrm{D}$ printed plate, a scanning electron microscope was used to obtain a detailed image of the sample and to analyze the distribution of $\mathrm{BaTiO} 3$ and PEGDA particles.

\section{RESULTS}

\section{A. Laser Doppler Vibrometer Results}

The measurements obtained using laser Doppler vibrometer method showed that the 3D printed samples presented in this paper behave as thin plates. Two models exist in the literature to predict the modes of vibration of circular thin layers. The model of a membrane considers a thin layer only dependent on tension, while the model of the plate is only dependent on stiffness. Furthermore, a membrane resonance frequency is inversely proportional to its thickness, while a plate is directly proportional to it [26]. While fabricating the samples, it become clear that a thicker sample had a higher resonance frequency and therefore a thin plate model was chosen (see Eq. 7). As shown in Figure 7a for PEGDA 250 and in Figure 7b for PEGDA 700, it can be clearly seen that the plates present several modes of vibration, both radial and circular modes. It is possible to predict the frequency of the modes of vibration of a circular plate as reported in [26], where modes $(1,1)$ and $(0,2)$ are predicted to be respectively 2.091 and 3.909 times higher than mode $(0,1)$. The $3 \mathrm{D}$ printed plates present all the modes of vibrations predicted by [26] and their frequencies are in good agreement with those predicted in the literature.

Table I shows the average resonance frequency for the two types of PEGDA samples. These values were useful while modeling the plates with the transfer matrix method, as they were used as initial guess to initiate the optimization procedure to retrieve the other parameters.

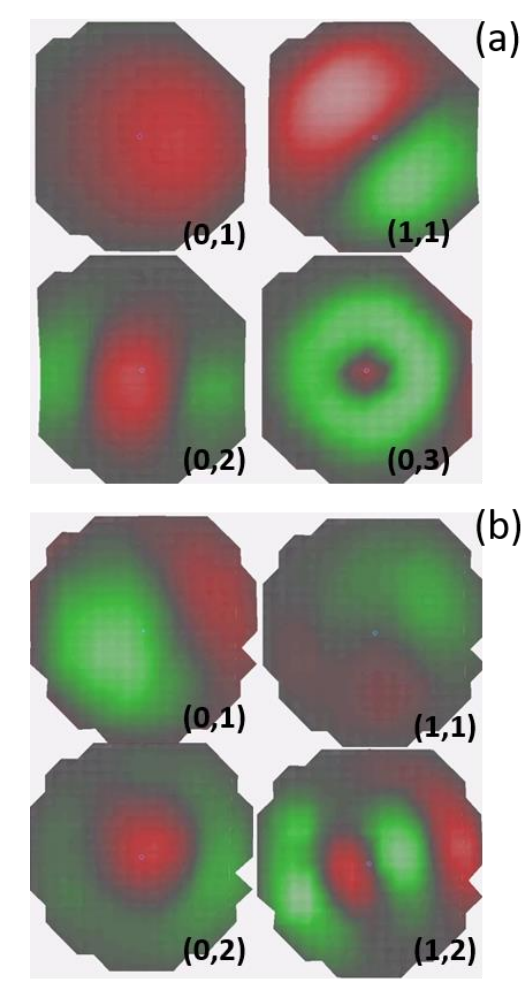

Figure 7. Modes $(0,1),(1,1),(0,2),(0,3)$ for the PEGDA 250 sample (a). Modes $(0,1),(1,1),(0,2),(1,2)$ for the PEGDA 700 sample (b).

TABLE I

MATERIAL PROPERTIES - LDV

\begin{tabular}{llll}
\hline \hline Symbol & Quantity & $\begin{array}{l}\text { PEGDA } \\
250\end{array}$ & $\begin{array}{l}\text { PEGDA } \\
700\end{array}$ \\
\hline$f_{0}(0,1)$ & $\begin{array}{l}\text { Fundamental } \\
\text { frequency }(\mathrm{Hz})\end{array}$ & 940 & 400 \\
$(1,1)$ & $\begin{array}{l}\text { First radial mode } \\
(\mathrm{Hz})\end{array}$ & 1500 & 1070 \\
& $\begin{array}{l}\text { First circular mode } \\
(\mathrm{Hz})\end{array}$ & 2300 & 1530 \\
\hline \hline
\end{tabular}

Values of fundamental frequency and first circular and radial modes for samples of PEGDA 250 and PEGDA 700 having a $15 \mathrm{~mm}$ diameter.

\section{B. Impedance Tube Results}

The transmission and reflection coefficients were first retrieved through impedance tube measurements and are represented respectively by the solid magenta and green lines in Figure 8 for PEGDA 250 (a) and 700 (b). The same coefficients together with absorption were then retrieved using the transfer matrix model and initialized using the values given by laser Doppler vibrometer for the resonance frequency and by vendors data sheets and literature values for density, Young's modulus, Poisson's ratio and loss factor. To minimize the difference between transmission, reflection and absorption coefficients obtained experimentally with those obtained analytically, the 
values of density, Young's modulus, Poisson's ratio and loss TABLE II

MATERIAL PROPERTIES - IMPEDANCE TUbE

\begin{tabular}{llll}
\multicolumn{4}{c}{ MATERIAL PROPERTIES - IMPEDANCE TUBE } \\
\hline \hline & & $\begin{array}{l}\text { PEGDA } \\
250\end{array}$ & $\begin{array}{l}\text { PEGDA } \\
700\end{array}$ \\
& & & \\
\hline$E$ & Youngntity & 3.04 & 0.67 \\
$\rho$ & Density $\left(\mathrm{kg} / \mathrm{m}^{3}\right)$ & 1730 & 2170 \\
$\eta$ & Loss & 0.070 & 0.075 \\
$v$ & Poisson's Ratio & 0.35 & 0.35 \\
$\mathrm{f}_{0}$ & Fundamental Frequency & 827 & 346 \\
& (Hz) & & \\
\hline \hline
\end{tabular}

Properties of the materials used to 3D print the plates as retrieved by using the transfer matrix approach and optimization with the impedance tube measurements.
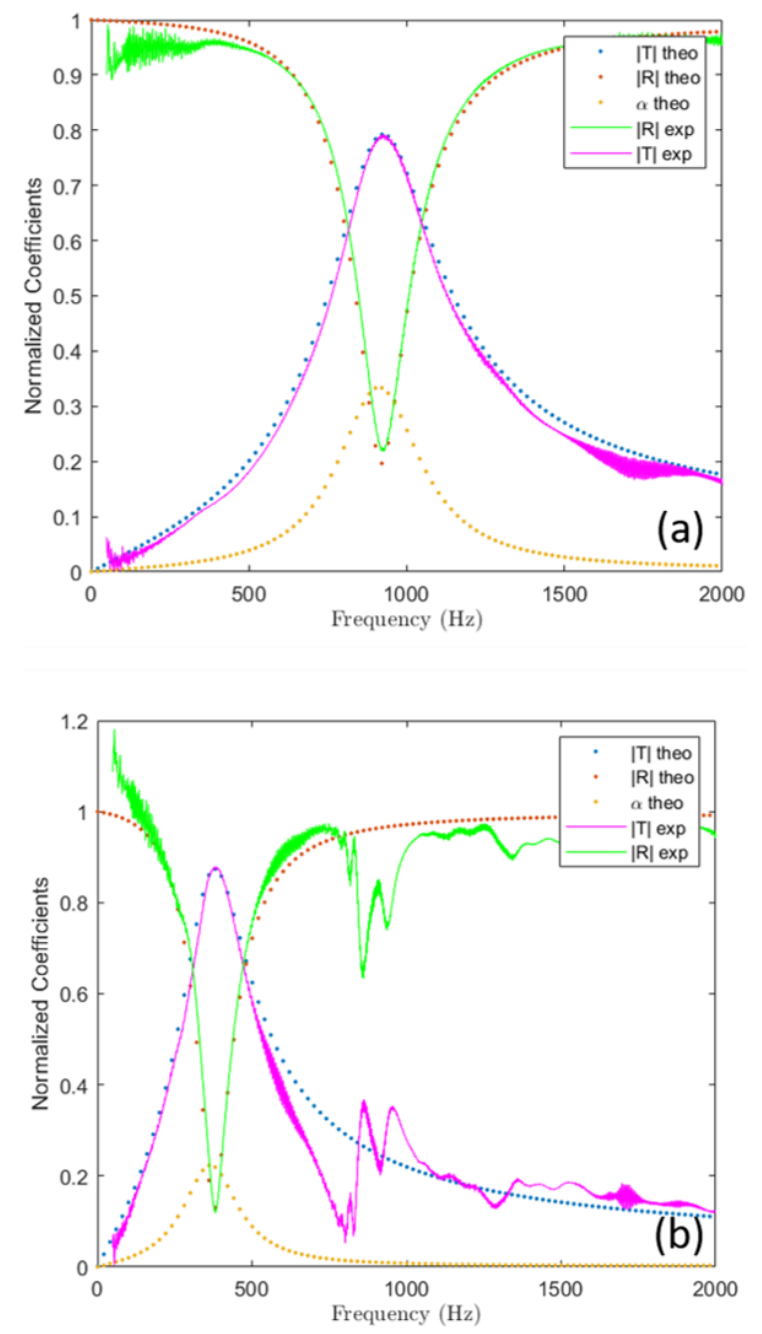

Fig. 8. Transmission, reflection and absorption coefficients represented by the dotted lines correspond to the parameters retrieved with the optimized transfer matrix model. The solid lines show reflection and transmission coefficients measured through the impedance tube and normalized to the input signal. PEGDA 250 values are shown in graph (a) and PEGDA 700 in graph (b). factor assumed at the modeling stage where changed iteratively during an optimization process, until the modeled coefficients graph in Figure 8 fit into the graph representing the experimental coefficients. Therefore, the density, Young's modulus, Poisson's ratio and loss factor parameters retrieved in this section result from the process of minimization of the difference between experimental coefficients obtained through measurements in the impedance tube and theoretical transmission, reflection and absorption coefficients modeled with the transfer matrix approach. Through this inverse method it was therefore possible to obtain the values of Young's modulus, density, Poisson's ratio, loss factor and fundamental frequency listed in Table II. The differences between the values of fundamental frequency given by laser vibrometer and the impedance tube approach might be due to the differences in samples preparation. In the first case, the plate is in the free field and its support is glued to a piece of glass. Hence, a tiny volume of air is created between the plate and the support. In the second case, there is no enclosed volume of air underneath the plate, which is in a tube instead than the free field. The values retrieved through LDV measurements should therefore be used just as initial values for our model and to show the presence of the modes in the plates. Figure 8 shows that the plates fabricated in this work would be suitable for acoustic metamaterials applications. In fact, both kinds of samples show a high reflection coefficient hence low transmission before the first mode of resonance, which corresponds to a region of negative effective density [16], [31], [32].

\section{Nanoindentation Results}

Nanoindentation measurements were gathered on both top and bottom sides of the samples. By looking at Figure 9, it can be seen that the particles distribution is different on the two sides of the plate. The bottom side tends to contain more BaTiO3 particles than the top side. Therefore, two different values for the Young's Modulus of both PEGDA 250 and PEGDA 700 samples were retrieved, as outlined in Table III. This nonhomogeneous distribution of particles in the plate can possibly explain the different values of Young's modulus given by nanoindentation and retrieved through impedance tube measurements. Since the tip used for nanoindentation is small in comparison to the dimension of the particles of PEGDA and $\mathrm{BaTiO} 3$ in the plate, it can be inferred that, for this specific type of plates, impedance tube measurements are more reliable than nanoindentation. Moreover, the sample required for nanoindentation must be small and cut from the support, hence possibly eliminating the small stress that the plate could have received while being attached to the support during the $3 \mathrm{D}$ printing process. This sample preparation process could cause a modification of the physical properties of the plate. Therefore, in future designs containing these plates, the results from impedance tube measurements will be preferred when modeling the system. 


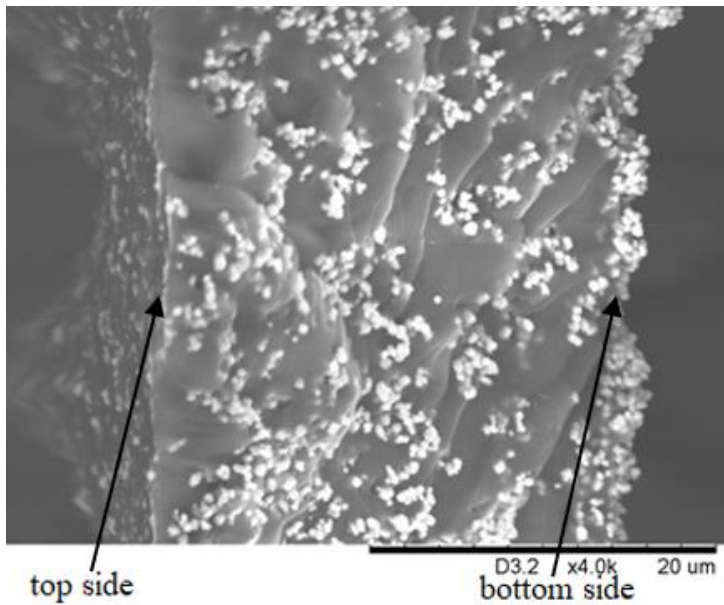

Fig. 9. Cross-section of the PEGDA 250 sample used for nanoindentation and obtained with scanning electron microscope. It can be noticed that the top and bottom side contain different amounts of BaTiO3 and PEGDA particles.

TABLE III

MATERIAL PROPERTIES - NANOINDENTATION

\begin{tabular}{llll}
\hline \hline Symbol & Quantity & $\begin{array}{l}\text { PEGDA } \\
250\end{array}$ & $\begin{array}{l}\text { PEGDA } \\
700\end{array}$ \\
\hline$E$ & $\begin{array}{l}\text { Young's Modulus } \\
\text { (GPa) Top }\end{array}$ & 0.30 & 0.062 \\
$E$ & $\begin{array}{l}\text { Young's Modulus } \\
\text { (GPa) Bottom }\end{array}$ & 0.12 & 0.059 \\
\hline \hline
\end{tabular}

Young's modulus of PEGDA 250 and PEGDA 700 obtained through nanoindentation measurements. Both top and bottom of the samples have been measured because of non-homogeneous distribution of BaTiO3 in the sample, as highlighted by scanning electron microscopy.

\section{CONCLUSION}

Thin flat plates were successfully $3 \mathrm{D}$ printed using innovative stereolithography techniques based on direct light processing. Thanks to these new techniques it was possible to carefully choose suitable materials and engineer a thin plate that could be used to attenuate low frequencies for example in road traffic, engine or aircraft noise. Recently this goal has been achieved by exploiting active noise cancellation in headphones, and in the future acoustic metamaterials could be integrated in electroacoustic devices or inside cars and aircrafts to achieve a similar noise attenuation. Other frequency ranges can be attenuated by using different materials or thicker plates. Three different characterization techniques were used, namely laser Doppler vibrometer, nanoindentation and impedance tube with transfer matrix model to retrieve the physical quantities and parameters related to the plate under test. In particular, impedance tube measurements confirmed to be a suitable and complete technique for this kind of materials. Laser Doppler vibrometer was also very useful to quickly identify the vibration modes and it could be employed to retrieve the physical parameters if used in conjunction with a simulation technique such as finite element analysis. Nanoindentation was less preferred, in that the tip is very small and could randomly sample a part of the plate where BaTiO3 is not present. The obtained physical parameters will be integrated in future designs where the plates will be incorporated in more complicated structures. The fabrication technique presented in this paper could be scaled to cover different frequency ranges and for larger structures, for example to attenuate noise in the field of aerospace and other transportation industries. Nevertheless, this specific design would be more suitable for small-scale applications, such as headphones, smart speakers and other electroacoustic devices.

\section{ACKNOWLEDGMENT}

The authors would like to thank Dr. Milovan Cardona for his help in performing nanoindentation and in using the scanning electron microscope at the University of Strathclyde Biomedical Engineering Department. This article is based upon work from COST Action DENORMS CA15125, supported by COST (European Cooperation in Science and Technology) and by the European Research Council under the European Union's Seventh Framework Programme (FP/2007-2013) / ERC Grant Agreement n. [615030].

[8] Z. Y. Yang, W. Wen, P. Sheng, "Sound attenuating structures,"

[9] 7.395,898, 2008. metamaterial panels for sound attenuation in the $50-1000 \mathrm{~Hz}$ regime," Appl. Phys. Lett., 2010.

[10] S. H. Lee, C. M. Park, Y. M. Seo, Z. G. Wang, and C. K. Kim, "Acoustic metamaterial with negative density," Phys. Lett. A, vol. 373, no. 48, pp. 4464-4469, 2009.

[11] S. Xiao, G. Ma, Y. Li, Z. Yang, and P. Sheng, "Active control of membrane-type acoustic metamaterial by electric field," Appl. Phys. Lett., 2015.

[12] C. J. Naify, C.-M. Chang, G. McKnight, and S. Nutt, "Transmission loss and dynamic response of membrane-type locally resonant acoustic metamaterials," J. Appl. Phys., vol. 108, no. 11, p. 114905, Dec. 2010.

[13] C. J. Naify, C.-M. Chang, G. McKnight, and S. Nutt, "Transmission loss of membrane-type acoustic metamaterials with coaxial ring masses," J. Appl. Phys., vol. 110, no. 12, p. 124903, Dec. 2011.

[14] C. J. Naify, C.-M. Chang, G. McKnight, F. Scheulen, and S. Nutt, 
"Membrane-type metamaterials: Transmission loss of multi-celled arrays," J. Appl. Phys., vol. 109, no. 10, p. 104902, May 2011.

[15] F. Langfeldt, J. Riecken, W. Gleine, and O. von Estorff, "A membrane-type acoustic metamaterial with adjustable acoustic properties," J. Sound Vib., vol. 373, pp. 1-18, 2016.

[16] N. Sui, X. Yan, T.-Y. Huang, J. Xu, F.-G. Yuan, and Y. Jing, "A lightweight yet sound-proof honeycomb acoustic metamaterial," Appl. Phys. Lett., vol. 106, no. 17, p. 171905, Apr. 2015.

[17] C. J. Naify, C.-M. Chang, G. McKnight, and S. R. Nutt, "Scaling of membrane-type locally resonant acoustic metamaterial arrays," $J$. Acoust. Soc. Am., vol. 132, no. 4, pp. 2784-2792, Oct. 2012.

[18] H. Gong, M. Beauchamp, S. Perry, A. T. Woolley, and G. P. Nordin, "Optical Approach to Resin Formulation for 3D Printed Microfluidics.," RSC Adv., vol. 5, no. 129, pp. 106621-106632, Dec. 2015

[19] B. H. Song and J. S. Bolton, "A transfer-matrix approach for estimating the characteristic impedance and wave numbers of limp and rigid porous materials," J. Acoust. Soc. Am., vol. 107, no. 3, pp. 1131-1152, Feb. 2000.

[20] A. Geslain, J.-P. Groby, V. Romero-García, F. Cervera, and J. Sánchez-Dehesa, "Acoustic characterization of silica aerogel clamped plates for perfect absorption," J. Non. Cryst. Solids, vol. 499, pp. 283-288, 2018.

[21] M. R. Stinson, "The propagation of plane sound waves in narrow and wide circular tubes, and generalization to uniform tubes of arbitrary cross-sectional shape," J. Acoust. Soc. Am., vol. 89, no. 2, pp. 550-558, Feb. 1991.

[22] N. Jiménez, J.-P. Groby, V. Pagneux, and V. Romero-García, "Iridescent Perfect Absorption in Critically-Coupled Acoustic Metamaterials Using the Transfer Matrix Method," Applied Sciences, vol. 7, no. 6. 2017.

[23] A. Merkel, G. Theocharis, O. Richoux, V. Romero-García, and V. Pagneux, "Control of acoustic absorption in one-dimensional scattering by resonant scatterers," Appl. Phys. Lett., vol. 107, no. 24, p. 244102, Dec. 2015.

[24] J.-P. Groby, W. Huang, A. Lardeau, and Y. Aurégan, "The use of slow waves to design simple sound absorbing materials," J. Appl. Phys., vol. 117, no. 12, p. 124903, Mar. 2015.

[25] F. Bongard, H. Lissek, and J. R. Mosig, "Acoustic transmission line metamaterial with negative/zero/positive refractive index," Phys. Rev. B, vol. 82, no. 9, p. 94306, Sep. 2010.

[26] P. M. C. Morse, Vibration and Sound. McGraw-Hill Book Company, 1948

[27] F. P. Mechel, "Formulas of Acoustics," Noise Control Eng. J., 2009.

[28] D. T. Blackstock, Fundamentals of physical acoustics. John Wiley \& Sons, 2000.

[29] M. Niskanen et al., "Deterministic and statistical characterization of rigid frame porous materials from impedance tube measurements," J. Acoust. Soc. Am., vol. 142, no. 4, pp. 2407-2418, Oct. 2017.

[30] W. C. Oliver and G. M. Pharr, "An improved technique for determining hardness and elastic modulus using load and displacement sensing indentation experiments," J. Mater. Res., vol 7, no. 6, pp. 1564-1583, 1992

[31] S. Y. and X. Z. and G. Hu, "Investigation of the negative-mass behaviors occurring below a cut-off frequency," New J. Phys., vol. 12, no. 10, p. 103025, 2010.

[32] S. Varanasi, J. S. Bolton, T. H. Siegmund, and R. J. Cipra, "The low frequency performance of metamaterial barriers based on cellular structures," Appl. Acoust., vol. 74, no. 4, pp. 485-495, 2013.

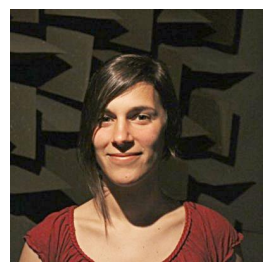

Cecilia Casarini ( $\left.\mathrm{S}^{\prime} 17\right)$ is a $\mathrm{PhD}$ student in Electronic \& Electrical Engineering within the Centre for Ultrasonic Engineering at the University of Strathclyde in Glasgow, UK. Her doctoral research focusses on 3D printed acoustic metamaterials for applications to noise and sound control in electroacoustic devices such as hearing aids and headphones. In 2018 she was and Audio and Acoustic Engineering Intern in the Acoustics Hardware team at Apple,
Inc. in Cupertino, CA. She received a MSc degree in Acoustics and Music Technology in 2016 at the University of Edinburgh, UK.

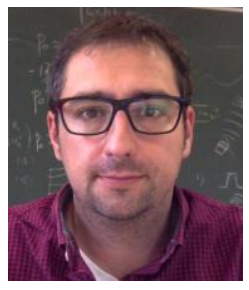

Vicente Romero-García is CNRS researcher at the Acoustic Laboratory of Le Mans University (LAUM, UMR CNRS 6613) since 2014. His research interests cover a wide range of subjects in wave physics. In particular wave propagation in periodic media as phononic and sonic crystals, random media, complex structures as well as acoustic metamaterials, and metasurfaces.

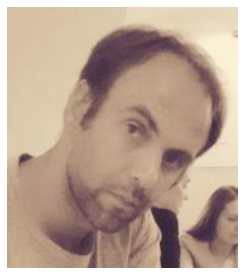

Jean-Philippe Groby is CNRS researcher at the Acoustic Laboratory of Le Mans University (LAUM, UMR CNRS 6613) since 2009. His research interests cover the design, characterization and application of complex structures for the control of audible sound, e.g. metamaterials, meta surfaces,

and metafluid.

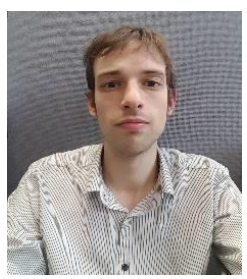

Benjamin Tiller is a Postdoctoral researcher in the Department of Electronic and Electrical Engineering at the University of Strathclyde, Glasgow, United Kingdom. $\mathrm{He}$ graduated from the University of Nottingham with an MSc degree in Physics and then received his $\mathrm{PhD}$ degree in Biomedical Engineering from University of Glasgow in 2016. His $\mathrm{PhD}$ research focused on the Physics of acoustic streaming in microfluidic channels, and phononic sensors. His current research interests focus on 3D printing of functional materials and novel acoustic sensors.

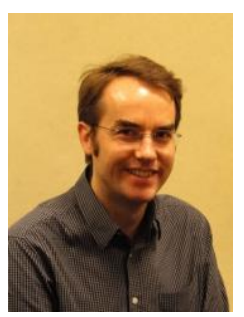

James F. C. Windmill (M'99-SM'17) is a Professor in the Department of Electronic and Electrical Engineering at the University of Strathclyde, Glasgow, United Kingdom. $\mathrm{He}$ has over 18 years of research and development experience in the areas of sensors and hearing systems. His research interests are in the field of biologicallyinspired acoustic systems, from the fundamental biology to various engineering application topics.

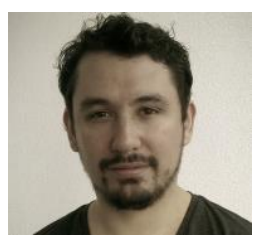

Joseph C. Jackson is a Lecturer in Electronic and Electrical Engineering at the University of Strathclyde, based in the Centre for Ultrasonic Engineering. His research interests cover a wide range of subjects, such as the physical basis for hearing, sound production and reception in biology and engineering, and bio-inspired transducer design. 